\title{
Resveratrol inhibits mucus overproduction and MUC5AC expression in a murine model of asthma
}

\author{
ZHEN-HUA NI $^{1 *}$, JI-HONG TANG ${ }^{2 *}$, GUO CHEN $^{2}$, YI-MIN LAI ${ }^{2}$, QING-GE CHEN ${ }^{2}$, \\ ZAO $\mathrm{LI}^{2}$, WEI YANG ${ }^{2}, \mathrm{XU}-\mathrm{MIN} \mathrm{LUO}^{2}$ and XIONG-BIAO WANG ${ }^{2}$ \\ ${ }^{1}$ Central Laboratory; ${ }^{2}$ Department of Respiratory Medicine, Putuo Hospital, \\ Shanghai University of Traditional Chinese Medicine, Shanghai 200062, P.R. China
}

Received December 21, 2014; Accepted September 16, 2015

DOI: $10.3892 / \mathrm{mmr} .2015 .4520$

\begin{abstract}
Previous in vitro studies have demonstrated that resveratrol is able to significantly inhibit the upregulation of mucin 5AC (MUC5AC), a major component of mucus; thus indicating that resveratrol may have potential in regulating mucus overproduction. However, there have been few studies regarding the resveratrol-mediated prevention of MUC5AC overproduction in vivo, and the mechanisms by which resveratrol regulates MUC5AC expression have yet to be elucidated. In the present study, an ovalbumin (OVA)-challenged murine model of asthma was used to assess the effects of resveratrol treatment on mucus production in vivo. The results demonstrated that resveratrol significantly inhibited OVA-induced airway inflammation and mucus production. In addition, the mRNA and protein expression levels of MUC5AC were increased in the OVA-challenged mice, whereas treatment with resveratrol significantly inhibited this effect. The expression levels of murine calcium-activated chloride channel (mCLCA)3, an important key mediator of MUC5AC production, were also reduced following resveratrol treatment. Furthermore, in vitro studies demonstrated that resveratrol significantly inhibited human (h)CLCA1 and MUC5AC expression in a dose-dependent manner. These results indicated that resveratrol was effective in preventing mucus overproduction and MUC5AC expression in vivo, and its underlying mechanism may be associated with regulation of the mCLCA3/hCLCA1 signaling pathway.
\end{abstract}

Correspondence to: Dr Xiong-Biao Wang, Department of Respiratory Medicine, Putuo Hospital, Shanghai University of Traditional Chinese Medicine, 164 Lanxi Road, Shanghai 200062, P.R. China

E-mail: xiongbiao6@hotmail.com

${ }^{*}$ Contributed equally

Key words: resveratrol, asthma, mucosal, mucin 5AC, murine CLCA3, human CLCA1

\section{Introduction}

Asthma is one of the most common chronic inflammatory diseases worldwide. The prevalence of asthma has increased significantly in recent decades, and it currently affects $\sim 300$ million individuals worldwide (1). It is estimated that the number of people diagnosed with asthma will increase to 400 million by 2025 (2). Further research regarding asthma is essential, since certain symptoms, including mucus overproduction, are currently incurable.

Mucus production has a key role in bronchial asthma, and contributes to the morbidity and mortality of the disease. There are $\sim 20$ mucin genes in humans, which are designated MUC followed by a number (3). Mucin 5AC (MUC5AC), is a major component of mucus that has been reported to have a key role in the process of mucus overproduction in asthma $(4,5)$. Various inflammatory cytokines, including tumor necrosis factor (TNF)- $\alpha$, bacterial products, growth factors and chemicals are able to activate transcription factors, such as specificity protein 1 (Sp1), cAMP response element-binding protein (CREB), and nuclear factor- $\kappa \mathrm{B}$, which may induce the expression of MUC5AC mRNA and protein in airway epithelial cells (6). Nakanishi et al (6) demonstrated that the introduction of human calcium-activated chloride channel (hCLCA1) into human NCI-H292 cells induced mucus production and MUC5AC expression. In addition, Kang et al (7) reported that a bidirectional circuit between the Notch and epidermal growth factor receptor signaling pathways was able to regulate MUC5AC expression. The transcriptional mechanism that regulates MUC5AC/Muc5ac expression is well-defined; however, there are currently no satisfactory treatments for mucus overproduction. At present, glucocorticoids are used most frequently to reduce mucus production; however, their effects are not always optimal.

Resveratrol (trans-3,5,4'-trihydroxystilbene) is a polyphenol that is present in numerous types of plants, including grapes. Resveratrol exhibits a wide range of biological and pharmacological activities, such as anticarcinogeneic, cardiovascular protective and anti-inflammatory activities. Previous studies have reported on the anti-asthmatic effects of resveratrol in murine models of asthma. Treatment with resveratrol has been shown to inhibit the levels of T helper (Th)2 cytokines interleukin (IL)-4 and IL-5, airway hyperresponsiveness and 
eosinophilic infiltration $(8,9)$. In addition, in vitro studies have demonstrated that benzo(a)pyrene (10), epidermal growth factor, phorbol 12-myristate 13-acetate and TNF- $\alpha$-induced MUC5AC upregulation and mucin production were inhibited by resveratrol in NCI-H292 human bronchial epithelium cells (11). These results indicated the potential application of resveratrol in regulating mucus overproduction. However, there have been few studies regarding resveratrol-mediated prevention of MUC5AC overproduction in vivo, and the mechanisms by which resveratrol regulate MUC5AC expression have yet to be elucidated.

Therefore the present study aimed to assess the expression levels of MUC5AC and its upstream genes in a murine model of asthma following treatment with resveratrol. The results demonstrated that resveratrol was effective in preventing mucus overproduction and inhibiting MUC5AC expression in vivo.

\section{Materials and methods}

Animals. Female BALB/c mice (8-12 weeks-old) were purchased from Sino-British SIPPR/BK Lab Animal Ltd. (Shanghai, China). All experimental procedures complied with the International Standards of Animal Welfare, and were approved by the Institute Animal Care and Use Committee of Shanghai University of Traditional Chinese Medicine (Shanghai, China).

Ovalbumin (OVA)-induced murine model of asthma, and resveratrol treatment. A total of 30 female BALB/c mice were divided into three groups: The normal control group (saline-challenged mice treated with saline; $n=10 /$ group), the asthma group (OVA-challenged mice treated with saline; $n=10 /$ group), and the resveratrol group (OVA-challenged mice treated with resveratrol; $n=10 /$ group). The murine asthma model was established as previously described (12). Briefly, all mice from the asthma and resveratrol groups were immunized by intraperitoneal (IP) injection with $100 \mu \mathrm{g}$ OVA (Sigma-Aldrich, St. Louis, MO, USA) complexed with alum on days 0 and 15, whereas the mice in the control group received an injection of saline. The mice in the asthma and resveratrol groups received an intranasal dose of $100 \mu \mathrm{g}$ OVA on days 15 , 26, 27 and 28, whereas the mice in the control group received saline. Between days 29 and 35, the resveratrol group was injected with $30 \mathrm{mg} / \mathrm{kg}$ resveratrol (IP; Sigma-Aldrich) once a day according to the method of Lee et al (7); the control and asthma groups received saline.

On day 36 , the mice from all groups were sacrificed by cervical dislocation. The chest was opened using surgical scissors, and the lungs were removed from the thoracic cavity by careful dissection and were washed with saline. The left lungs were collected for histological analyses, and the right lungs were collected for RNA extraction.

Lung histopathology. The left lungs of the mice were harvested and fixed with $2 \mathrm{ml}$ of $10 \%$ neutral-buffered formalin (Sigma-Aldrich). Once fixed in neutral-buffered formalin for $72 \mathrm{~h}$, the lung tissues were embedded in paraffin, and cut into 4- $\mu \mathrm{m}$ serial sections using a microtome (Leica RM2235; Leica Biosystems Nussloch GmbH, Nußloch, Germany). The tissue sections were stained with hematoxylin and eosin (H\&E) solution (Beyotime Institute of Biotechnology, Haimen, China), in order to evaluate airway inflammation; and periodic acid schiff (PAS) solution (Shanghai Sunbio Medical Biotechnology Co., Ltd., Shanghai, China), in order to assess mucus production, according to the manufacturer's protocol. Tissue sections were observed and images were captured using an optical microscope (DM300; Leica, Wetzlar, Germany). The degree of airway inflammatory cell infiltration was evaluated according to the Underwood scoring system (13) on a scale of $0-4(0$, none; 1 , mild; 2 , moderate; 3 , marked; and 4 , severe). Goblet cell hyperplasia in airway epithelium was evaluated by the following scoring system: $0,<5 \%$ goblet cells; $1,5-25 \% ; 2,25-50 \% ; 3,50-75 \%$; and $4,>75 \%$, as outlined by Lee et al (8). The scores were measured in a double-blind manner by two independent investigators.

Immunohistochemistry for MUC5AC and murine (m)CLCA3 detection. Immunohistochemistry was conducted as described previously (14). Tissue sections from the left lungs of the mice were deparaffinized in xylene, and rehydrated in graded ethanol. Sections were boiled at a constant temperature of $95^{\circ} \mathrm{C}$ for $15 \mathrm{~min}$ in citrate buffer $(0.01 \mathrm{mmol} / \mathrm{l}$; $\mathrm{pH}$ 6.0; Fuzhou Maixin Biotechnology Development Co., Ltd., Fuzhou, China) for antigen retrieval. The tissue sections were then incubated with biotin-labeled mouse monoclonal anti-mouse MUC5AC (cat.no. ab79082; diluted 1:200) or rabbit polyclonal anti-mouse mCLCA3 (cat. no. ab46512; diluted 1:4000) antibodies (both Abcam, Cambridge, UK) at $4^{\circ} \mathrm{C}$ overnight. Subsequently, the tissue sections were washed three times with phosphate-buffered saline (PBS) and incubated for $30 \mathrm{~min}$ with horseradish peroxidase (HRP)-Streptavidin (cat. no. 43-8323; diluted 1:1,000; Invitrogen; Thermo Fisher Scientific, Inc., Waltham, MA, USA) or HRP-conjugated anti-rabbit immunoglobulin (Ig)G secondary antibody (cat. no. k4002; Dako Corp., Glostrup, Denmark). Tissue sections were then washed three times with PBS and stained with diaminobenzidine (Fuzhou Maixin Biotechnology Development Co., Ltd., Fuzhou, China). Images were captured using a Leica DM300 microscope (DM300; Leica). Image-Pro Plus 6.0 software (Media Cybernetics, Inc., Rockville, MD, USA) was used to measure the area and integrated optical density (IOD) of the stained slides, and the staining intensity was determined by mean IOD (mean IOD=IOD/area).

Reverse transcription-quantitative polymerase chain reaction ( $q P C R)$. Total RNA was isolated from the right lung tissues of the mice using TRIzol ${ }^{\circledR}$ reagent (Invitrogen; Thermo Fisher Scientific, Inc.) as described previously (12). First-strand cDNA was prepared using random hexamer primers provided with the SuperScriptIII ${ }^{\mathrm{TM}}$ First-Strand Synthesis kit (Invitrogen; Thermo Fisher Scientific, Inc.), according to the manufacturer's protocol. qPCR was performed using a Universal Master mix (Roche Diagnostics, Indianapolis, IN, USA) on an ABI Real-Time PCR system (Applied Biosystems; Thermo Fisher Scientific, Inc.). The primer sequences (Sangon Biotech Co., Ltd., Shanghai, China) are listed in Table I. Each assay was performed in triplicate. The PCR cycling conditions used for all reactions were as follows: $10 \mathrm{~min}$ at $95^{\circ} \mathrm{C}$, followed by 40 two-step cycles at $95^{\circ} \mathrm{C}$ for $15 \mathrm{sec}$ and $60^{\circ} \mathrm{C}$ for $1 \mathrm{~min}$. The relative expression levels of the target genes were normalized against glyceraldehyde 3-phosphate dehydrogenase and analyzed using REST2009 software (14). 
Table I. Sequences of primers and probes.

\begin{tabular}{|c|c|c|}
\hline Primer or probe & GenBank number & Sequence \\
\hline GADPH-F & NM_001289726.1 & 5'-AGGTCGGTGTGAACGGATTTG-3' \\
\hline GADPH-R & & 5'-TGTAGACCATGTAGTTGAGGTCA-3' \\
\hline MUC5AC-F & NM_010844.1 & 5'-CAGGACTCTCTGAAATCGTACCA-3' \\
\hline MUC5AC-R & & 5'-GAAGGCTCGTACCACAGGG-3' \\
\hline CLCA3-F & NM_017474.2 & 5'-CGTCATCGCCATAGACCACG-3' \\
\hline CLCA3-R & & 5'-GCCTCGTATATTCAGGCTTTGC-3' \\
\hline KLF4-F & NM_010637.3 & 5'-GTGCCCCGACTAACCGTTG-3' \\
\hline KLF4-R & & 5'-GTCGTTGAACTCCTCGGTCT-3' \\
\hline HES1-F & NM_008235.2 & 5'-CCAGCCAGTGTCAACACGA-3' \\
\hline HES1-R & & 5'-AATGCCGGGAGCTATCTTTCT-3' \\
\hline TSTA3-F & NM_031201.1 & 5'-AAGGTGGTTGCAGATGGGG-3' \\
\hline TSTA3-R & & 5'-CCATTGCAGCGAGATGGATGA-3' \\
\hline LRRFIP2-F & NM_001164838.1 & 5'-CATTGTCTCTTCGGAGCCTTG-3' \\
\hline LRRFIP2-R & & 5'-GGATGACTGCCCACTTAGAGG-3' \\
\hline KRAS-F & NM_021284.6 & 5'-CAAGAGCGCCTTGACGATACA-3' \\
\hline KRAS-R & & 5'-CCAAGAGACAGGTTTCTCCATC-3' \\
\hline RPS6KA3-F & NM_148945.2 & 5'-ATGGATGAACCTATGGGAGAGG-3' \\
\hline RPS6KA3-R & & 5'-AAGCTGTCTAGCATCAGAGCC-3' \\
\hline CTSC-F & NM_009982.4 & 5'-CAACTGCACCTACCCTGATCT-3' \\
\hline CTSC-R & & 5'-TAAAATGCCCGGAATTGCCCA-3' \\
\hline PLA2G4A-F & NM_008869.3 & 5'-CAGCACATTATAGTGGAACACCA-3' \\
\hline PLA2G4A-R & & 5'-AGTGTCCAGCATATCGCCAAA-3' \\
\hline
\end{tabular}

F, forward; R, reverse.

Cell culture. The 16HBE human lung epithelial cell line (Bogoo Biotech Co., Ltd., Shanghai, China) was cultured in RPMI-1640 medium supplemented with $10 \%$ fetal bovine serum (Invitrogen). Briefly, $1 \times 10^{6}$ cells were seeded into a $100-\mathrm{mm}$ dish and incubated overnight. After $24 \mathrm{~h}$, the 16HBE cells were treated with 10 or $50 \mu \mathrm{mol} / 1$ resveratrol for $72 \mathrm{~h}$. An equivalent volume of vehicle (dimethyl sulfoxide; Sigma-Aldrich) was added to the control cells. Following a $72-\mathrm{h}$ incubation at $37^{\circ} \mathrm{C}$, the cells were harvested for western blotting.

Western blot analysis. Western blotting was conducted as described previously (15). Total protein was isolated from the cells using ProteoJET ${ }^{\mathrm{TM}}$ Mammalian Cell Lysis Reagent (Fermentas; Thermo Fisher Scientific, Inc., Pittsburgh, PA, USA). The protein fractions ( $40 \mu \mathrm{g}$ per lane) were separated by $10 \%$ sodium dodecyl sulfate-polyacrylamide gel electrophoresis, and then transferred to a poly vinylidene fluoride (PVDF) membrane (EMD Millipore, Bedford, MA, USA). The PVDF membrane was blocked with $5 \%$ bovine serum albumin (Sigma-Aldrich) and washed twice with Tris-buffered saline containing Tween (TBST; Beyotime Institute of Biotechnology). The membrane was then incubated with biotin-labeled mouse monoclonal anti-human MUC5AC (cat. no. ab79082; diluted 1:1,000; Abcam), anti-hCLCA1 (cat. no. 7072; diluted 1:1,000; Santa Cruz Biotechnology, Inc., Dallas, TX, USA) and rabbit monoclonal anti-human GAPDH (cat. no. 5174S; diluted 1:1,000; Cell Signaling Technology, Inc., Danvers, MA, USA) antibodies overnight at $4^{\circ} \mathrm{C}$, and washed three times with TBST. Subsequently, the membrane was incubated with HRP-Streptavidin (Invitrogen) or HRP-conjugated anti-mouse IgG secondary antibody (Cell Signaling Technology, Inc.) for $2 \mathrm{~h}$ at room temperature. Finally, immunoreactive bands were detected using enhanced chemiluminescence (EMD Millipore, Bedford, MA, USA).

Statistical analysis. SPSS version 16.0 software (SPSS, Inc., Chicago, IL, USA) was used for data analyses. Data are presented as the mean \pm standard deviation. Statistical significance was determined by Student's t-test or Mann-Whitney $\mathrm{U}$ test. $\mathrm{P}<0.05$ was considered to indicate a statistically significant difference.

\section{Results}

Effects of resveratrol on airway inflammation and mucus production in the lung tissue of a murine model of asthma. Histological analysis of the lung sections detected differences in inflammation between the various groups. Treatment with resveratrol reduced airway inflammation in the lung tissues, as compared with the asthma group (Fig. $1 \mathrm{~A}-\mathrm{C} ; \mathrm{P}=0.002$ ), thus suggesting that resveratrol exerted anti-inflammatory effects. In addition, PAS staining was used to evaluate mucus production. Mucus production in the asthma group was increased, as compared with the control group, whereas mucus production was decreased in the resveratrol-treated group, as compared with the asthma group (Fig. $2 \mathrm{~A}-\mathrm{C} ; \mathrm{P}=0.001$ ). These results were consistent with previous observations (8). These data 
A

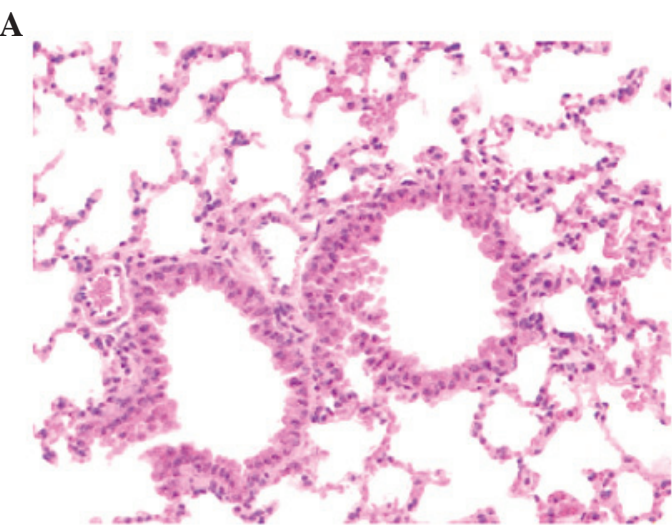

C .

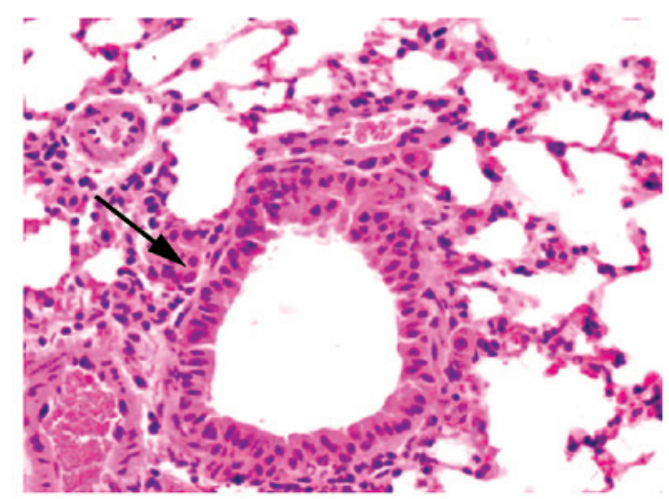

B

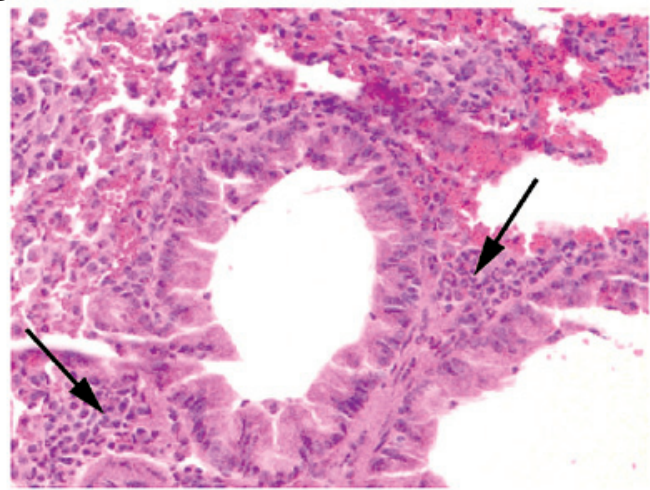

D

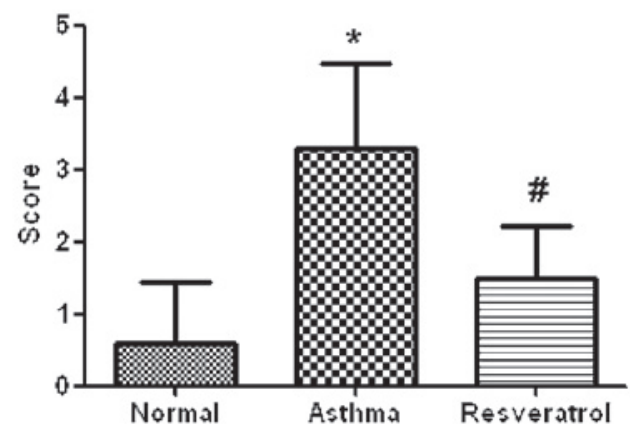

Figure 1. Effects of resveratrol on airway inflammation in a murine model of asthma. Airway inflammation was determined by hematoxylin and eosin staining (magnification, x200). (A) Saline control group (untreated); (B) asthma group (ovalbumin-challenged); and (C) resveratrol group. (D) Scoring of the extent of inflammation in the three groups. ${ }^{\#} \mathrm{P}=0.002$ vs. the asthma group; ${ }^{*} \mathrm{P}=0.001$ vs. the normal group. Inflammatory cells were indicated by an arrow.

A

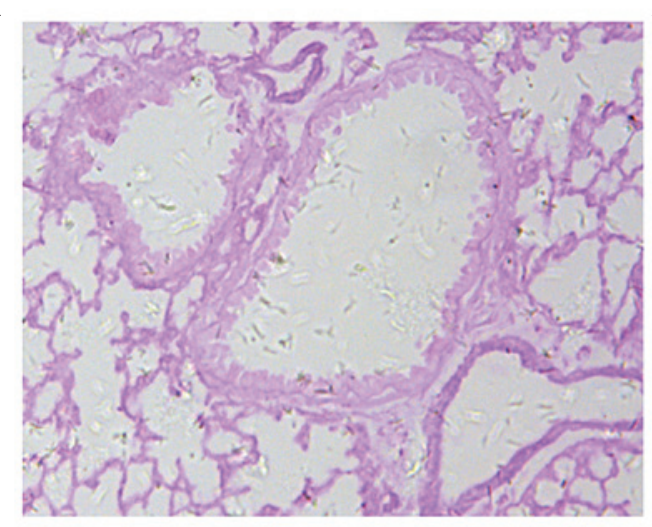

C

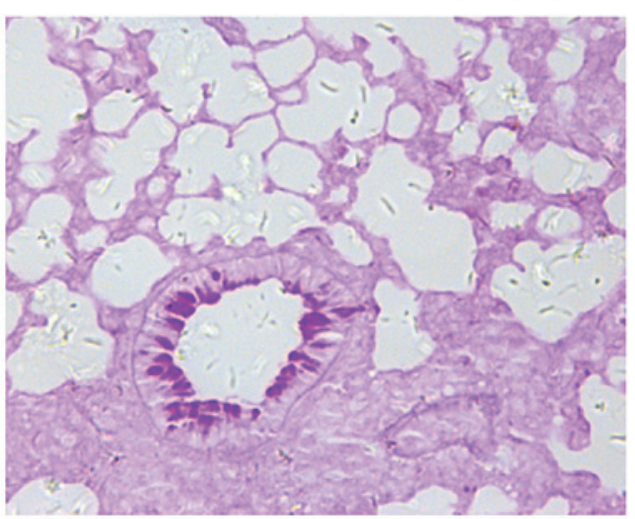

B

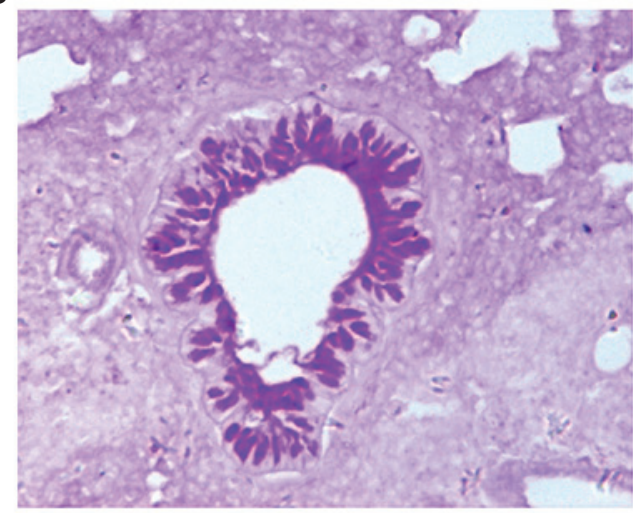

D

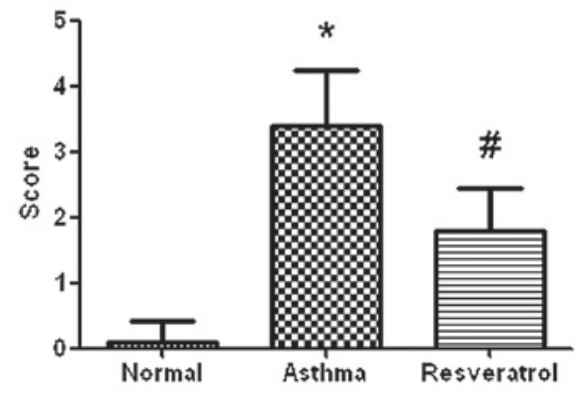

Figure 2. Effects of resveratrol on mucus production in a murine model of asthma. Mucus production was assessed using periodic acid schiff staining (magnification, x200). (A) Saline control group (untreated); (B) asthma group (ovalbumin-challenged); and (C) resveratrol group. (D) Scoring of the extent of mucus production in the three groups. ${ }^{*} \mathrm{P}=0.001$ vs. the asthma group; ${ }^{*} \mathrm{P}<0.001$ vs. the normal group. 
Table II. Relative expression of mucin 5AC (MUC5AC) and MUC5AC-associated genes, compared with the asthma group.

\begin{tabular}{|c|c|c|c|c|c|}
\hline \multirow[b]{2}{*}{ Gene } & \multirow{2}{*}{$\frac{\text { Asthma group }}{\text { Expression ratio }}$} & \multicolumn{2}{|c|}{ Normal group } & \multicolumn{2}{|c|}{ Resveratrol group } \\
\hline & & Expression ratio & P-value & Expression ratio & P-value \\
\hline MUC5AC & 1 & 0.014 & $<0.001$ & 0.131 & $<0.001$ \\
\hline CLCA3 & 1 & 0.002 & $<0.001$ & 0.041 & $<0.001$ \\
\hline KLF4 & 1 & 0.423 & 0.209 & 0.733 & 0.262 \\
\hline HES 1 & 1 & 0.529 & 0.199 & 1.297 & 0.269 \\
\hline TSTA3 & 1 & 0.576 & 0.086 & 0.906 & 0.564 \\
\hline LRRFIP2 & 1 & 0.386 & 0.091 & 1.813 & 0.269 \\
\hline KRAS & 1 & 0.555 & 0.219 & 1.306 & 0.272 \\
\hline RPS6KA3 & 1 & 0.452 & 0.080 & 0.985 & 0.944 \\
\hline CTSC & 1 & 0.396 & 0.046 & 0.988 & 0.949 \\
\hline PLA2G4A & 1 & 0.485 & 0.103 & 1.181 & 0.425 \\
\hline
\end{tabular}
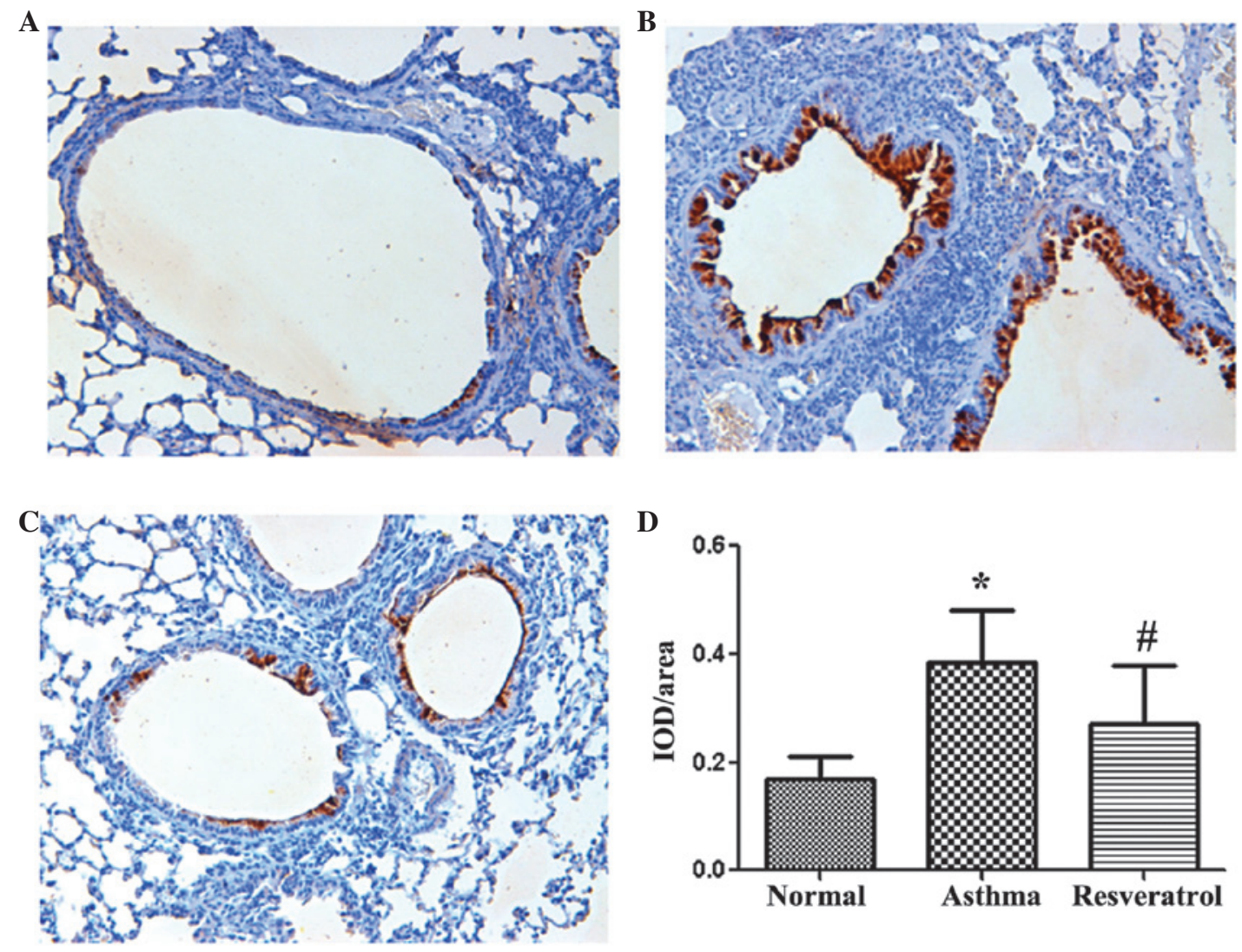

Figure 3. MUC5AC protein expression in the lungs. Immunohistochemical staining of MUC5AC in the lung tissue (magnification, x200). (A) Saline control group (untreated); (B) asthma group (ovalbumin-challenged); and (C) resveratrol group. (D) Mean IOD of MUC5AC staining. ${ }^{*} \mathrm{P}=0.022$ vs. the asthma group; ${ }^{*} \mathrm{P}<0.001$ vs. the normal group. MUC5AC, mucin 5AC; IOD, integrated optical density.

suggest that resveratrol may reduce airway inflammation and mucus production in a mouse model of asthma.

Effects of resveratrol on MUC5AC expression. MUC5AC is the major component of mucus in asthma among the products of 20 mucin genes (5), and the expression levels of MUC5AC may represent the intensity of mucin expression. To determine whether resveratrol-induced inhibition of mucus production was associated with reduced MUC5AC expression, MUC5AC protein expression was determined using immunohistochemistry. As shown in Fig. 3, MUC5AC expression was increased in the asthma group, as compared with the control group $(\mathrm{P}<0.001)$. Conversely, treatment with resveratrol significantly inhibited MUC5AC protein expression ( $\mathrm{P}=0.022$, vs. the asthma group). In addition, reduced mRNA expression levels of MUC5AC were detected in the resveratrol-treated mice, as compared with those in the OVA-challenged mice (Table II; $\mathrm{P}<0.001$ ). These data suggest 
A

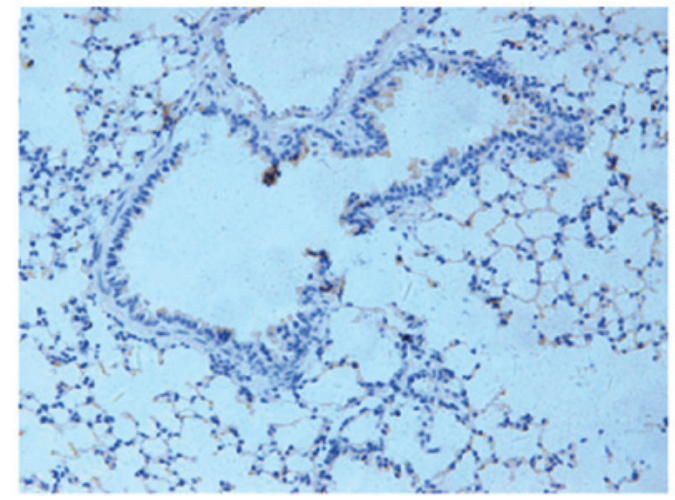

C

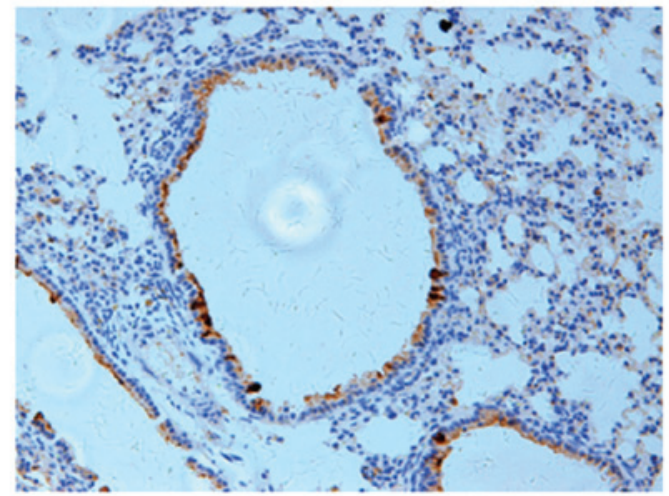

B

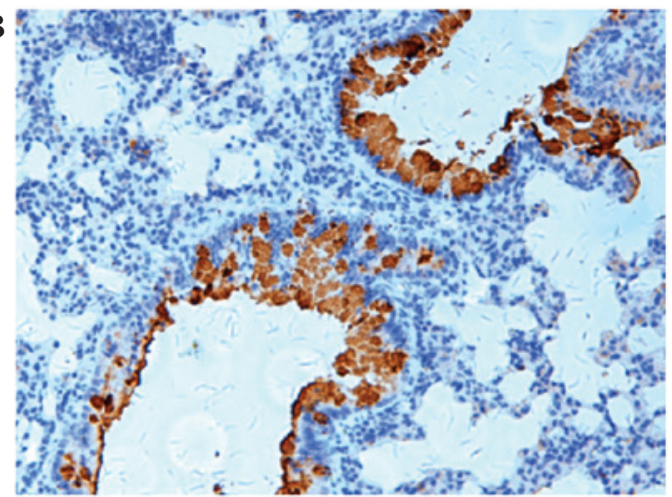

D

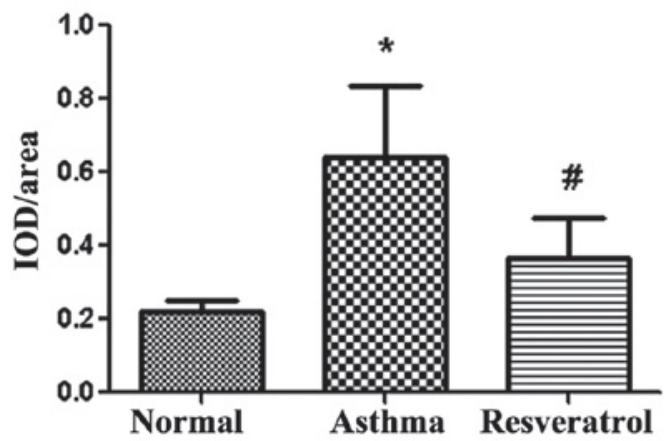

Figure 4. mCLCA3 protein expression in the lungs. Immunohistochemical staining of mCLCA3 in the lung tissue (magnification, $\mathrm{x} 200$ ). (A) Saline control group (untreated); (B) asthma group (ovalbumin-challenged group); and (C) resveratrol group. (D) Mean IOD of mCLCA3 staining. "P=0.001 vs. the asthma group; ${ }^{*} \mathrm{P}<0.001$ vs. the normal group. mCLCA3, murine calcium-activated chloride channel 3; IOD, integrated optical density.

that resveratrol may inhibit mucus production by regulating MUC5AC transcription.

Effects of resveratrol on the expression of nine MUC5AC-related genes. To explore the possible signal pathway underlying resveratrol-induced MUC5AC inhibition, nine MUC5AC-related genes were selected from the MUC5AC core gene list compiled by Wang et al (16). These genes were the key mediators of Wnt, Notch, mitogen-activated protein kinases and signal transducer and activator of transcription (STAT)6 pathways, and had been reported to affect the expression of MUC5AC. In the present study, two genes [mCLCA3and cathepsin C (CTSC)] were overexpressed in the asthma group, as compared with the control group (Table II; $\mathrm{P}<0.001$ for $\mathrm{mCLCA} 3$; $\mathrm{P}=0.046$ for $\mathrm{CTSC}$ ). However, only mCLCA3 mRNA expression was reduced following resveratrol treatment (Table II; $\mathrm{P}<0.001$ ). Consistent with these results, immunohistochemistry revealed that mCLCA3 protein expression was reduced in the resveratrol group, as compared with the asthma group (Fig. 4; $\mathrm{P}=0.001$ ).

Effects of resveratrol on hCLCA1 and MUC5AC expression in a lung epithelial cell line. To examine whether resveratrol could effectively inhibit hCLCA1 and MUC5AC expression in human cells, the 16HBE human lung epithelial cell was treated with 10 or $50 \mu \mathrm{mol} / 1$ resveratrol for $72 \mathrm{~h}$. Resveratrol markedly inhibited hCLCA1 and MUC5AC protein expression in a dose-dependent manner (Fig. 5). These results confirmed the effects of resveratrol on regulating CLCA expression.

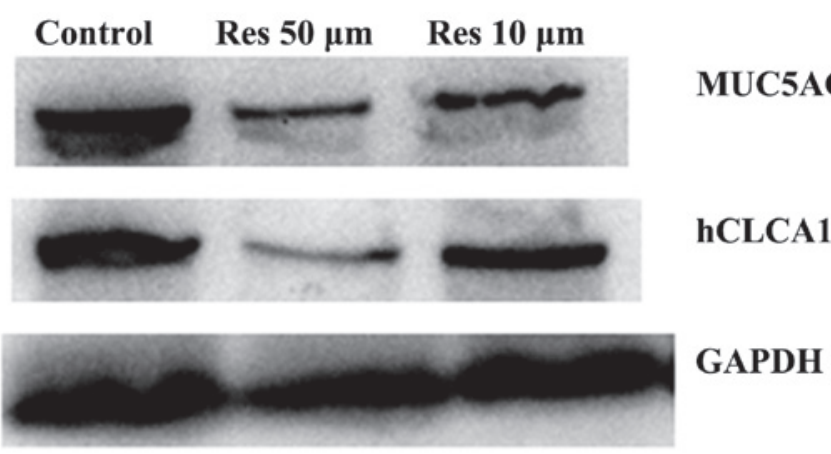

Figure 5. Detection of hCLCA1 and MUC5AC protein expression in $16 \mathrm{HBE}$ human lung epithelial cells by western blotting. The cells were treated with 10 or $50 \mu \mathrm{mol} / 1$ resveratrol for $72 \mathrm{~h}$. MUC5AC, mucin $5 \mathrm{AC}$; hCLCA1, human calcium-activated chloride channel 1; GAPDH, glyceraldehyde 3-phosphate dehydrogenase.

\section{Discussion}

Mucus hypersecretion is a common symptom of asthma, and is an important factor in asthma-associated mortality. The present study demonstrated that resveratrol was an effective reagent in a murine model of asthma, particularly by suppressing mucus production. In addition, it was suggested that the effects of resveratrol on mucus production may be associated with the downregulation of MUC5AC overexpression, via the mCLCA3/hCLCA1 signaling pathway.

The CLCA family consists of numerous members. The hCLCA subfamily has four members: hCLCA1, hCLCA2, 
hCLCA3 and hCLCA4, whereas the Mus musculus subfamily has at least five members. hCLCA1, an ortholog of the murine CLCA3, has an important role in mucus hypersecretion (17). Overexpression of hCLCA1 in human NCI-H292 cells has previously been demonstrated to induce mucus production and MUC5AC expression (6). Consistent with these results, mCLCA3 antibodies may significantly reduce MUC5AC expression (18). In addition, the protein and mRNA expression levels of mCLCA3 and MUC5AC were significantly increased in lung tissue when mCLCA3 plasmids were transfected into the airways of normal BALB/c mice (19). In the airway hyperresponsiveness mouse model, inhibiting mCLCA3 expression by intra-tracheal administration of an adenovirus expressing mCLCA3 antisense RNA efficiently suppressed the asthma phenotype and mucus overproduction (6). Therefore, targeting hCLCA1/mCLCA3 may be an effective method for the modulation of mucus production.

CLCA protein is secreted from cells, bound to the CLCA receptor, which stimulates an increase in MUC5AC and MUC5B expression, which in turn leads to enhanced mucin secretion (20). Therefore, it may be hypothesized that decreasing mCLCA3 expression is at least one of the mechanisms by which resveratrol inhibits MUC5AC expression. However, the mechanism by which resveratrol modulates mCLCA3 expression remains unclear. The authors of the present study hypothesize that regulation of mCLCA3 may be either direct or indirect. It has previously been reported that mCLCA 3 was upregulated by Th2 cytokines (IL-4, IL-9 and IL-13) in a murine model of asthma, possibly via Janus kinase (JAK)/STAT6 signaling $(21,22)$. Previous studies have demonstrated that resveratrol reduced the levels of IL-4 and IL-13 in asthmatic mice $(8,23)$; therefore, it is possible that resveratrol may decrease mCLCA3 expression by inhibiting the expression of Th2 cytokines. Concurrently, resveratrol may prevent phosphorylation of JAK or inhibit the expression of JAK-1 directly, and thereby inhibit STAT6 phosphorylation (24). The mouse CLCA and human CLCA gene promoter regions contain consensus STAT6-binding sites. It has previously been demonstrated that hCLCA1 may be regulated by the JAK/STAT6 signaling pathway in humans (20). Furthermore, resveratrol has been shown to inhibit extracellular signal-regulated kinases (ERK)1/2 activation (25), and it has been suggested that hCLCA1 may be regulated by ERK signaling pathways (20). Therefore, there may be numerous signaling pathways underlying the resveratrol-mediated regulation of CLCA expression.

In conclusion, the results of the present study demonstrated that resveratrol may effectively suppress mucus overproduction in a murine model of asthma. The decreased mucus production was mediated partially by the regulation of mCLCA3/hCLCA1 gene expression, which consequently downregulated MUC5AC expression. Further research is required to determine the signaling pathway underlying resveratrol-mediated mCLCA3 regulation.

\section{Acknowledgements}

The authors of the present study would like to thank the Animal Research Center of Putuo Hospital, Shanghai University of Traditional Chinese Medicine. The present study was supported by the Shanghai Science and Technology Committee (nos. 10411969100 and 12401900404), the Shanghai Educational Committee (grant no. 13ZZ096), the Shanghai Putuo District Science and Technology Committee (no. 2009PTKW002), the NFSC project (no. 81402988), and the '085' Project (no. 085ZY1216).

\section{References}

1. Anandan C, Nurmatov U, van Schayck OCP and Sheikh A: Is the prevalence of asthma declining? Systematic review of epidemiological studies. Allergy 65: 152-167, 2010.

2. To T, Stanojevic S, Moores G, Gershon AS, Bateman ED, Cruz AA and Boulet LP: Global asthma prevalence in adults: Findings from the cross-sectional world health survey. BMC Public Health 12: 204, 2012.

3. Evans CM, Kim K, Tuvim MJ and Dickey BF: Mucus hypersecretion in asthma: Causes and effects. Curr Opin Pulm Med 15: 4-11, 2009.

4. Voynow JA, Young LR, Wang Y, Horger T, Rose MC and Fischer BM: Neutrophil elastase increases MUC5AC mRNA and protein expression in respiratory epithelial cells. Am J Physiol 276: L835-L843, 1999.

5. Izuhara K, Ohta S, Shiraishi H, Suzuki S, Taniguchi K, Toda S, Tanabe T, Yasuo M, Kubo K, Hoshino T and Aizawa H: The mechanism of mucus production in bronchial asthma. Curr Med Chem 16: 2867-2875, 2009.

6. Nakanishi A, Morita S, Iwashita H, Sagiya Y, Ashida Y, Shirafuji H, Fujisawa Y, Nishimura $\mathrm{O}$ and Fujino M: Role of gob-5 in mucus overproduction and airway hyperresponsiveness in asthma. Proc Natl Acad Sci USA 98: 5175-5180, 2001.

7. Kang JH, Lee EH, Park SW and Chung IY: MUC5AC expression through bidirectional communication of Notch and epidermal growth factor receptor pathways. J Immunol 187: 222-229, 2011.

8. Lee M, Kim S, Kwon OK, Oh SR, Lee HK and Ahn K: Anti-inflammatory and anti-asthmatic effects of resveratrol, a polyphenolic stilbene, in a mouse model of allergic asthma. Int Immunopharmacol 9: 418-424, 2009.

9. Royce SG, Dang W, Yuan G, Tran J, El Osta A, Karagiannis TC and Tang ML: Resveratrol has protective effects against airway remodeling and airway hyperreactivity in a murine model of allergic airways disease. Pathobiol Aging Age Relat Dis 1, 2011.

10. Chiba T, Uchi H, Tsuji G, Gondo H, Moroi Y and Furue M: Arylhydrocarbon receptor (AhR) activation in airway epithelial cells induces MUC5AC via reactive oxygen species (ROS) production. Pulm Pharmacol Ther 24: 133-140, 2011.

11. Lee SY, Lee HJ, Sikder MA, Shin HD, Kim JH, Chang GT, Seok JH and Lee CJ: Resveratrol inhibits mucin gene expression, production and secretion from airway epithelial cells. Phytother Res 26: 1082-1087, 2012.

12. Ni Z, Tang J, Cai Z, Yang W, Zhang L, Chen Q, Zhang L and Wang X: A new pathway of glucocorticoid action for asthma treatment through the regulation of PTEN expression. Respir Res 12: 47, 2011.

13. Underwood S, Foster M, Raeburn D, Bottoms S and Karlsson JA: Time-course of antigen-induced airway inflammation in the guinea-pig and its relationship to airway hyperresponsiveness. Eur Respir J 8: 2104-2113, 1995.

14. Pfaffl MW, Horgan GW and Dempfle L: Relative expression software tool (REST) for group-wise comparison and statistical analysis of relative expression results in real-time PCR. Nucleic Acids Res 30: e36, 2002.

15. Ni Z, Tao K, Chen G, Chen Q, Tang J, Luo X, Yin P, Tang J and Wang X: CLPTM1L is overexpressed in lung cancer and associated with apoptosis. PLoS One 7: e52598, 2012.

16. Wang G, Xu Z, Wang R, Al-Hijji M, Salit J, Strulovici-Barel Y, Tilley AE, Mezey JG and Crystal RG: Genes associated with MUC5AC expression in small airway epithelium of human smokers and non-smokers. BMC Med Genomics 5: 21, 2012.

17. Patel AC, Brett TJ and Holtzman MJ: The role of CLCA proteins in inflammatory airway disease. Annu Rev Physiol 71: 425-449, 2009.

18. Song L, Liu D, Wu C, Wu S, Yang J, Ren F and Li Y: Antibody to mCLCA3 suppresses symptoms in a mouse model of asthma. PLoS One 8: e82367, 2013.

19. Mei L, He L, Wu SS, Zhang B, Xu YJ, Zhang ZX, Zhao JP and Zhang HL: Murine calcium-activated chloride channel family member 3 induces asthmatic airway inflammation independently of allergen exposure. Chin Med J (Engl) 126: 3283-3288, 2013. 
20. Winpenny JP, Marsey LL and Sexton DW: The CLCA gene family: Putative therapeutic target for respiratory diseases. Inflamm Allergy Drug Targets 8: 146-160, 2009.

21. Nakano T, Inoue H, Fukuyama S, Matsumoto K, Matsumura M, Tsuda M, Matsumoto T, Aizawa H and Nakanishi Y: Niflumic acid suppresses interleukin-13-induced asthma phenotypes. Am J Respir Crit Care Med 173: 1216-1221, 2006.

22. Matsunaga Y, Inoue H, Fukuyama S, Yoshida H, Moriwaki A, Matsumoto T, Matsumoto K, Asai Y, Kubo M, Yoshimura A and Nakanishi Y: Effects of a Janus kinase inhibitor, pyridone 6, on airway responses in a murine model of asthma. Biochem Biophys Res Commun 404: 261-267, 2011.
23. Aich J, Mabalirajan U, Ahmad T, Khanna K, Rehman R, Agrawal A and Ghosh B: Resveratrol attenuates experimental allergic asthma in mice by restoring inositol polyphosphate 4 phosphatase (INPP4A). Int Immunopharmacol 14: 438-443, 2012.

24. Yang CJ, Lin CY, Hsieh TC, Olson SC and Wu JM: Control of eotaxin-1 expression and release by resveratrol and its metabolites in culture human pulmonary artery endothelial cells. Am J Cardiovasc Dis 1: 16-30, 2011.

25. Tan Y and Lim LH: trans-Resveratrol, an extract of red wine, inhibits human eosinophil activation and degranulation. Br J Pharmacol 155: 995-1004, 2008. 